\title{
An optimized procedure for quantitative analysis of mitophagy with the mtKeima system using flow cytometry
}

Nathaniel J Winsor ${ }^{\ddagger}, 1,2$ iD, Samuel A Killackey ${ }^{\ddagger}, 2$, Dana J Philpott ${ }^{1}$ \& Stephen E Girardin*,1,2

${ }^{1}$ Department of Immunology, University of Toronto, Toronto, ON, Canada; ${ }^{2}$ Department of Laboratory Medicine \& Pathobiology, University of Toronto, Toronto, ON, Canada; *Author for correspondence: stephen.girardin@utoronto.ca; ${ }^{\ddagger}$ Authors contributed equally

BioTechniques 69: 249-256 (October 2020) 10.2144/btn-2020-0071

First draft submitted: 17 May 2020; Accepted for publication: 6 July 2020; Published online: 18 August 2020

\section{ABSTRACT}

Mitophagy is the process by which mitochondria are selectively targeted and removed via autophagic machinery to maintain mitochondrial homeostasis in the cell. Recently, flow cytometry-based assays that utilize the fluorescent mtKeima reporter system have allowed for quantitative assessment of mitophagy at a single-cell level. However, clear guidelines for appropriate flow cytometry workflow and downstream analysis are lacking and studies using flow cytometry in mtKeima-expressing cells often display incorrect and arbitrary binary mitophagic or nonmitophagic cutoffs that prevent proper quantitative analyses. In this paper we propose a novel method of mtKeima data analysis that preserves subtle differences present within flow cytometry data in a manner that ensures reproducibility.

\section{METHOD SUMMARY}

In this report we propose an outline for the transfection of mtKeima into a cell monolayer, cell preparation and downstream analysis using flow cytometry and associated software, with emphasis on a novel data processing technique.

\section{KEYWORDS}

flow cytometry assays $\bullet$ gating strategies $\bullet$ mitophagy $\bullet$ mtKeima $\bullet$ ratio gating

Mitochondria are energy-producing organelles that are crucial to the function of numerous tissue types in eukaryotes, including the heart, skeletal muscle and brain [1]. Mitophagy is the process by which select mitochondria are targeted, engulfed by autophagosomes and removed through lysosomal degradation [2]. Mitochondria targeted for removal are often depolarized or damaged to an extent severe enough to initiate targeting pathways, leading to their labeling and eventual clearance [2]. The balance of mitochondrial biogenesis and degradation is an essential component of cellular homeostasis that maintains the mitochondrial population's performance at a level high enough to support the energy demands of the cell [3]. Defects in mitophagy and mitochondrial function in general have been connected to a broad group of illnesses including cardiovascular, neurodegenerative and metabolic diseases [3].

Broadly defined, mitophagy can be divided into two categories: the ubiquitin-mediated and mitophagy receptor-mediated pathways [4]. For many years the in vitro study of mitophagy revolved around the ubiquitin-mediated pathway, and in particular the PINK1-parkin mechanism [5]. Within this pathway, the retention and stabilization of PINK1 on the surface of depolarized mitochondria leads to sequential labeling with phosphorylation and ubiquitination prior to autophagosomal recruitment [5]. However, subsequent analysis has revealed additional pathways of targeting that function independently from PINK1 and parkin-mediated ubiquitination [6,7]. These alternate pathways involve 'mitophagy receptors': proteins in the outer mitochondrial membrane that can interact with and recruit MAP1A/MAP1B LC3-containing autophagosomes to mitochondria [4]. The relative importance of each receptor is likely dependent on the context of mitophagy induction; this remains an active area of investigation, although there is known to be functional overlap between receptors. Notably, the various pathways converge at the level of light chain 3 (LC3) recruitment for autophagophore targeting, allowing the mitochondrial cargo to be transported to the acidic environment of the lysosome for degradation and recycling [4]. Quantifying key steps in these pathways has become accepted practice for studying mitophagy. These events include parkin and LC3 recruitment to mitochondria, LC3I to LC3II conversion, the relative presence of mitochondria in the acidic environment of the lysosome compared with the more neutral cytosol, and lastly the removal of mitochondrial proteins [8].

Quantifying the levels of endogenous and overexpressed parkin recruitment to mitochondria provides an easy way to measure defects at the beginning of the mitophagy pathway [9]. However, relying exclusively on parkin-mitochondria colocalization does not adequately facilitate dissection of potential mechanisms. For example, a block or deficiency at the removal step of mitophagy can lead to a buildup of parkin-labeled mitochondria, which would be indistinguishable from an increase in mitochondrial targeting at the beginning of the pathway. Similarly, groups often use overexpressed and tagged LC3 in order to easily measure colocalization of this construct with mitochondria [10]. However, by overexpressing a marker for degradation, it is reasonable to assume mitochondria will be targeted more readily 
than in a cell with endogenous levels, biasing the data toward an apparently mitophagic state. The conversion of LC3I to its lipidated form (known as LC3II) has been a standard readout of autophagy in general [11]. The subset of autophagosomes involved in mitophagy are marked by LC3II recruitment to mitochondria [5]. However, there has been a shift away from overreliance on this readout due to the temporal and degradative nature of autophagic pathways [12]. As this is a removal process, a lack of LC3II targeted to mitochondria could simply reflect an increase in lysosomal clearance for the end stage of mitophagy. One solution is to block the final degradation step by preventing lysosomal acidification [12]. However, because we cannot specifically prevent lysosomal degradation of mitochondria without affecting removal of other cargo, preventing acidification can alter the cell in additional confounding ways, thereby complicating mechanistic studies $[13,14]$.

While it is obvious that these assays are best used in combination, there is still a need for accurate quantification of mitophagy at a single cell level. To this end, multiple groups have developed mitochondrial-localized fluorescent reporter systems that provide a snapshot of the mitochondrial population [15,16]. By comparing levels of free cytosolic mitochondria versus those in acidic lysosomes, a measure of the mitophagic state of each cell can be determined. These systems include lysosomal LAMP1-GFP (green fluorescent protein) colocalizing with mitochondrial markers, or alternatively mito-QC, a mitochondrial reporter that fluoresces differently at pHsensitive wavelengths $[17,18]$. In-depth discussions of these techniques have been reported previously; here we will focus on the mtKeima system [17-20]. Keima is a coral-derived, acid-stable fluorescent protein which can be excited by different wavelengths depending on the acidity of the environment [21]. By combining Keima with a certain localization sequence, the construct is localized to the mitochondrial matrix (mtKeima) and can be used as an indicator of the mitochondrial environment [15,21].

Numerous groups have utilized the mtKeima system in combination with fluorescent microscopy to obtain a visual representation of mitophagy [15,22]. Imaging software can then be used to quantify relative excitation with different laser wavelengths, giving a readout of the mitochondrial environment. This technique may have its greatest impact when studying mtKeima mice, as it allows researchers to visualize mitophagic activity within an organ of interest [20]. However, microscopy methods are better suited for qualitative analysis, or binary quantification, such as the proportion of colocalized puncta and their location within a given cell. Variation in staining, visualization and downstream analysis pipelines can all influence analysis of mitophagic activity and may result in the loss of valuable information, particularly when studying subtle shifts in mitophagy. Additionally, quantifying microscopic data usually entails manual cell-by-cell analysis. This is not only time intensive but by definition implies that only a selected sample of the cells will be analyzed, providing the opportunity for interpretative differences within and among labs. Due to the quantitative nature of flow cytometry and the ability of the technology to detect more subtle, gradient-like changes, several groups have recently combined the mtKeima system with flow cytometry $[15,16,23]$.

Unlike microscopy, flow cytometry is designed to accurately quantify large populations of diverse cell types. While this technique can reduce the bias and variability present in mitophagy analysis, there is currently no consensus as to how to design, process and analyze the samples $[16,24,25]$. A majority of studies employing mtKeima flow cytometry analyze the data by gating on the nonmitophagic population, in order to encompass a given percentage of the control treatment; this is termed 'binary gating' (Figure 1) [15,16,26]. Upon mitophagy treatment, cells outside of the control gate are deemed mitophagic, and a ratio of mitophagic to nonmitophagic cells can be derived.

While this method of analysis can yield statistically significant differences (Figures 1A, 1B \& 2C), it does so at the cost of important information. By binning cells into a binary 'mitophagic' or 'nonmitophagic' category, a cell that is only slightly above the threshold and one that is far beyond the gate will be weighted equally (Figures $1 \mathrm{~A} \& 2 \mathrm{C}$ ). Thus the natural spread of the data is collapsed.

Furthermore, some of these early flow cytometry assays are missing key quality control steps that are essential for well-controlled experiments, such as the use of a viability stain to discriminate dead or dying cells from a live, responsive population [23,25,27,28]. Viability stains provide increased ability to measure mitophagic responses in healthy or dying cells in a controlled manner, facilitating the visualization of these differences rather than relying on physical properties, such a forward and side scatter or adherence to a cell culture plate. While some groups use viability gating, it is not currently a standard across the field $[16,24,26]$.

\section{Materials \& methods}

\section{Cell transfection \& staining}

All experiments were performed with HEK293T cells, which endogenously express PINK1 and detectable levels of endogenous parkin [29,30]. Cells $\left(1 \times 10^{6} /\right.$ well) were transfected overnight with $4 \mu \mathrm{g} /$ well of pCHAC-mt-mKeima Red (Addgene Plasmid \#72342) at a ratio of 5:1 Fugene HD:plasmid (in $\mu \mathrm{g}$ ). Cells were then treated with carbonyl cyanide 3-chlorophenylhydrazone (CCCP; Sigma C2759) or Bafilomycin-A1 (Sigma B17983) for $20 \mathrm{~h}$. Media was aspirated and $500 \mu$ l of TrypLE Express cell dissociation reagent (Gibco 12604-013) added to each well, and the cells were incubated for $10 \mathrm{~min}$ at $37{ }^{\circ} \mathrm{C}, 5 \% \mathrm{CO}_{2}$. Cells were collected and diluted in phosphate-buffered saline (PBS; Wisen 311-425-CL) to inactivate cell dissociation reagent. Cells were pelleted and washed with PBS. Pellets were resuspended in $100 \mu$ of a viability stain (1:1000, Thermo Fisher, near-IR LIVE/DEAD L10119) for 10 min at room temperature, according to manufacturer's instructions. After staining, cells were pelleted and resuspended in $200 \mu$ of PBS and run immediately.

Cytometer settings \& gating

For all experiments presented here, cells were run on a BD Bioscience Fortessa X20 5 laser (355/405/488/561/640) flow cytometer equipped with Red 640 nm, Violet 405 nm and Yellow/Green 561 nm laser lines and Red 780/60, Violet 605/20 and Yellow/Green 


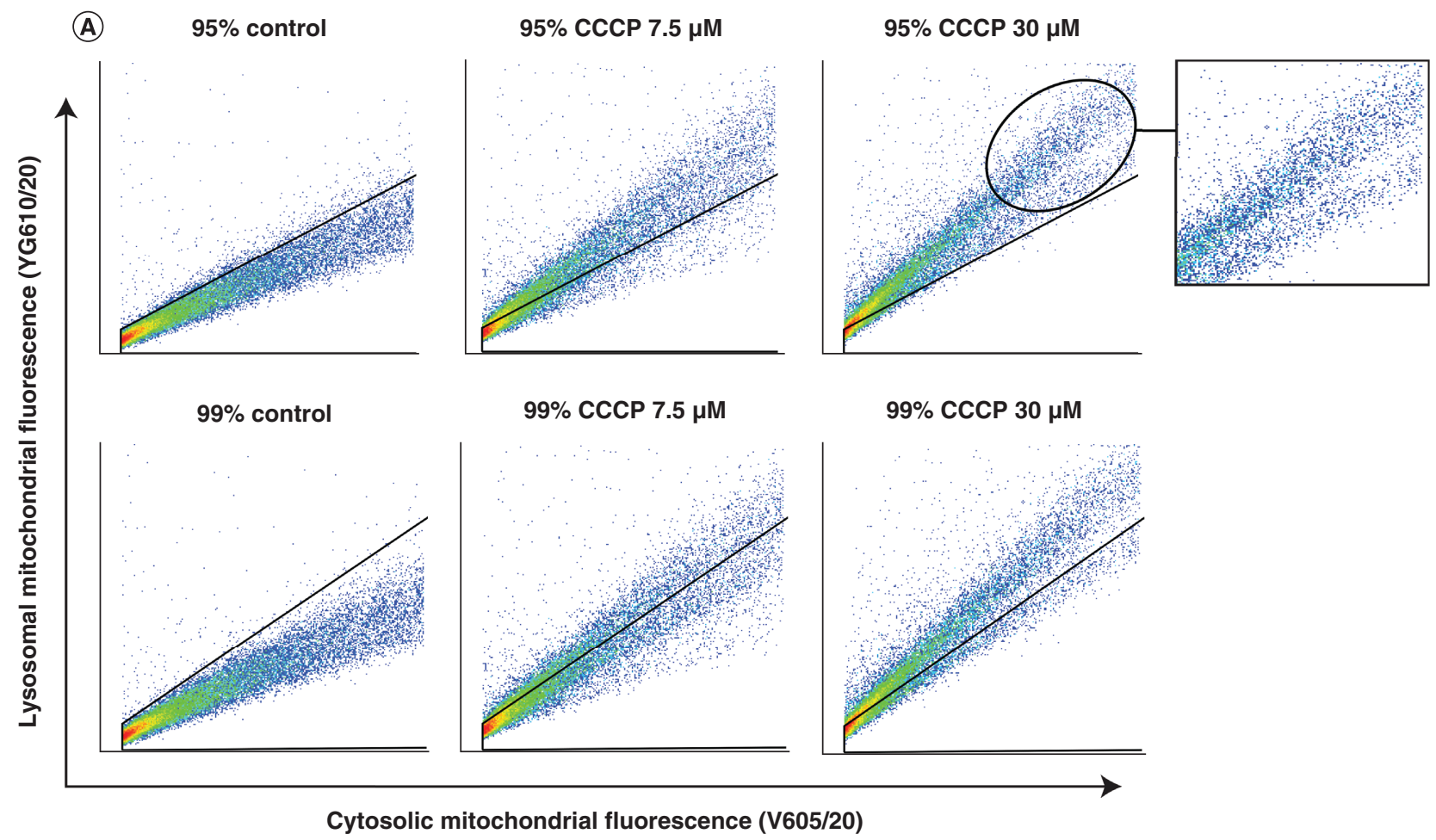

(B) $95 \%$ control gating

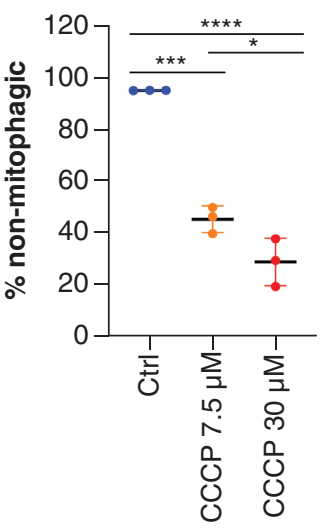

$95 \%$ control gating

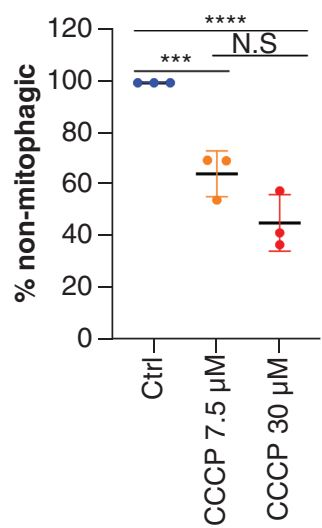

(C) $\mathrm{CCCP}$
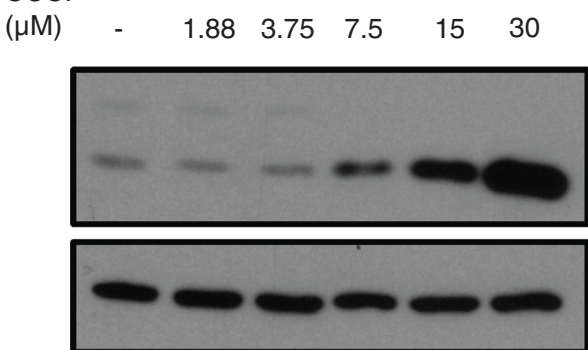

LC3

LC3II

Tubulin

Figure 1. Binary gating does not adequately capture shifts in mitophagic populations. Within the mtKeima system, a majority of cells express low levels of both cytosolic and lysosomal fluorescence. Binary gating is dependent on how this population is bisected by the investigator. As illustrated by gating the control population to include 95 versus $99 \%$ of nonmitophagic events (A), investigator choice can introduce statistically significant variability into data processing (B), which can obscure phenotypes under conditions that LC3 immunoblot demonstrate increased mitophagy (C). Additionally, binary gating can prevent analysis of mid to high mitophagic populations (inset, A), even while carrying a statistical difference (B).

$\mathrm{n}=3$, plotted with SD.

CCCP: Carbonyl cyanide 3-chlorophenylhydrazone; NS: Not significant.

$610 / 20$ detectors. Voltages were established using relevant stimulated (CCCP), unstimulated and unstained controls, to ensure that the voltage was sufficient to acquire data but not set so high as to force the majority of mtKeima+ events off-scale. Once determined in an initial experiment, calibration particles (Spherotech, URCP-38-2K) were utilized at the beginning of each flow cytometry session to ensure voltages were consistent $( \pm 10 \%)$ across previous replicates $[27,28]$. The gating strategy is outlined in Figure $3 \mathrm{~A}$. Cells were separated from debris by optical gating based on forward and side scatter area. Next, single cells were selected based on the ratio of forward scatter area to forward scatter height. Within the single cell population, live cells were defined by low LIVE/DEAD staining, when plotted against 

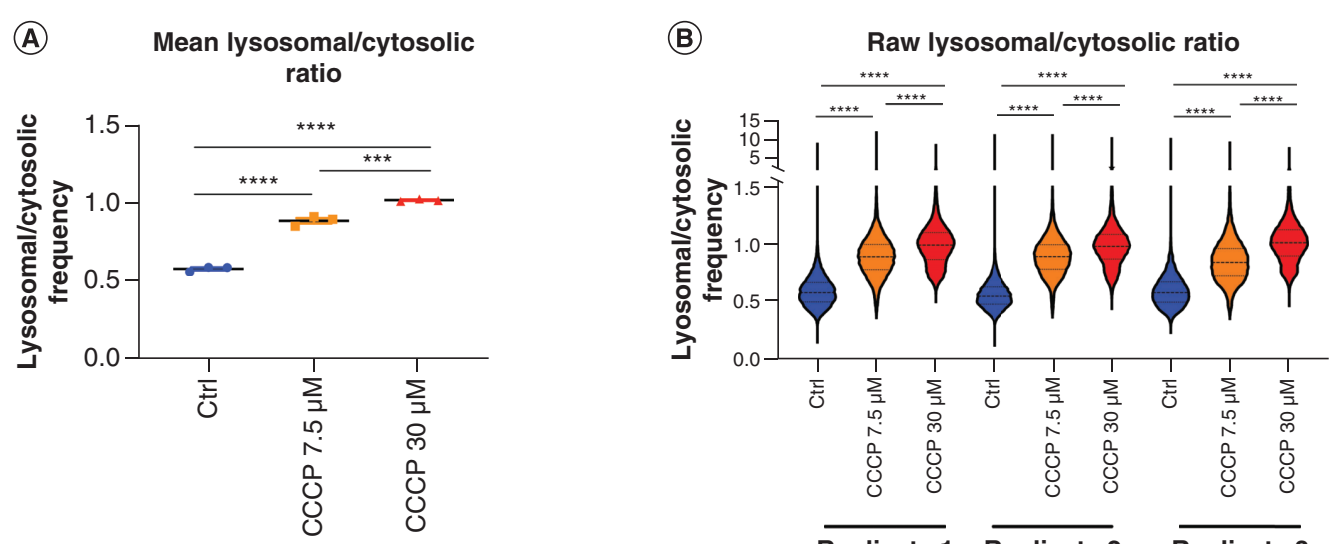

$\overline{\text { Replicate } 1} \overline{\text { Replicate } 2} \overline{\text { Replicate } 3}$

(C)

$$
95 \% \text { control }
$$

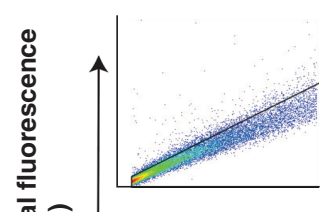

$99 \%$ control

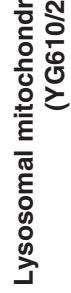

(D)
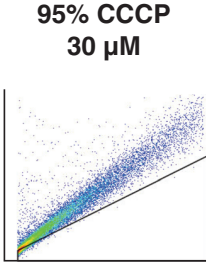

$99 \%$ CCCP $30 \mu \mathrm{M}$
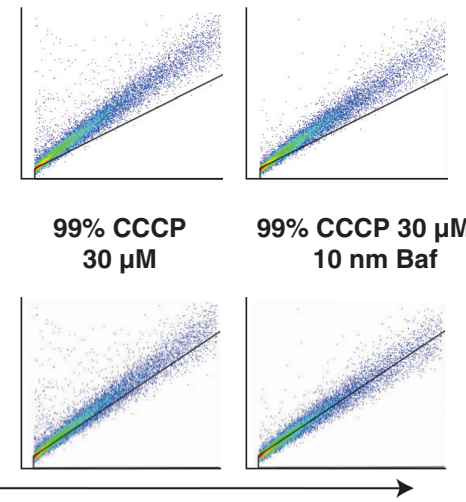
$10 \mathrm{~nm}$ Baf
Cytosolic mitochondrial fluorescence (V605/20)

$99 \% \operatorname{CCCP~} 30 \mu \mathrm{M}+$

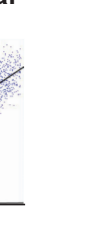

$95 \%$ control gating $\quad 99 \%$ control gating
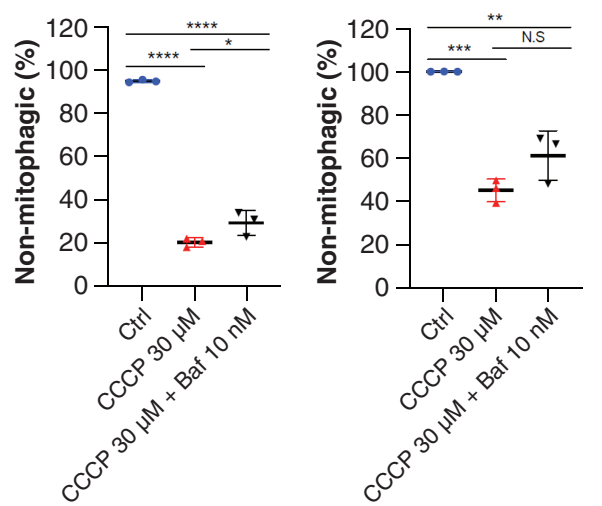

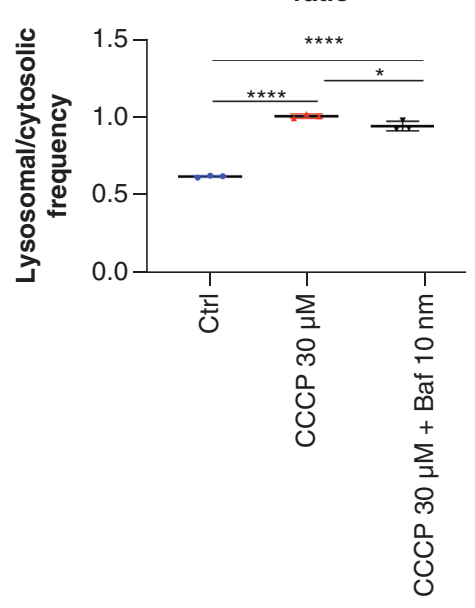

Figure 2. Ratio gating of lysosomal over cytosolic cell frequency is a repeatable, sensitive measure capable of detecting pharmacological dampening of mitophagy. The mean of the ratio gating strategy (A) is similar across replicates and is a phenocopy of LC3 immunoblotting (Figure 1C). (A) Ratio gating of 20,000 mtKeima + cells over three independent replicates. Violin plots (B) display standard deviation of each condition and are similar across replicates. (C) 20-h cotreatment of $30 \mu \mathrm{M} \mathrm{CCCP}$ and $10 \mathrm{nM}$ Baf results in abrogated induction of mitophagic populations, which is detectable by $95 \%$ but not $99 \%$ control gating. While $95 \%$ control gating is sufficient to detect the effect of Baf treatment, as shown in the representative plots, binary gating does not adequately capture middle and high mitophagic populations. In comparison, ratio gating (D) accurately and repeatably differentiates between control and treatment groups.

$\mathrm{n}=3$, plotted with SD (C) and SEM (B \& D).

Baf: Bafilomycin-A1; CCCP: Carbonyl cyanide 3-chlorophenylhydrazone. 


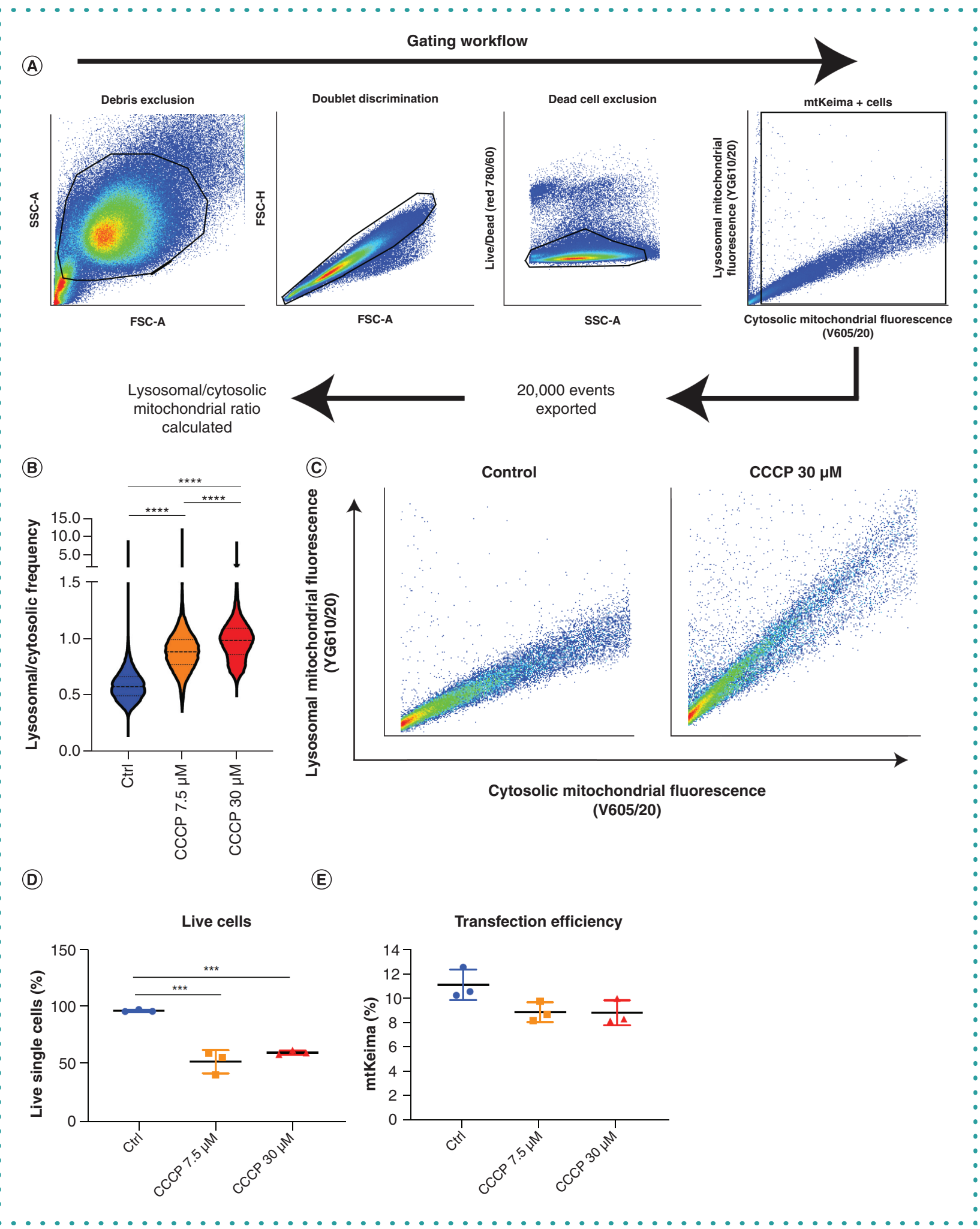

Figure 3. Proposed mtKeima workflow. (A) mtKeima + cells are first gated based on Forward (FSC-A) and Side Scatter (SSC-A) area, followed by doublet discrimination based on FSC height (FSC-H) by FSC-A. As mitophagic agents such as CCCP induce cell death (A \& D), only live cells are selected for downstream analysis as determined based on the viability stain. mtKeima+ cells are determined using untransfected controls (A). 20,000 scaled mtKeima + events were exported from FlowJo and the lysosomal/cytosolic ratio was calculated ((A), representative image in (B) and plots in (C)). CCCP induces an increase in lysosomal+ cells (B \& C). Transfection efficiency was similar across replicates but diminished on CCCP treatment (E). $\mathrm{n}=3$, plotted with SEM.

CCCP: Carbonyl cyanide 3-chlorophenylhydrazone; NS: Not significant. 
forward or side scatter area. As per established flow cytometry guidelines, all gating was based on relevant unstained (fluorescenceminus-one) populations [27,28]. Viability staining was confirmed through inclusion of a positive dead cell population, consisting of a 50:50 mixture of ethanol-treated and control cells. Within the live population, mtKeima+ cells were defined based on an untransfected control. Approximately 25,000 live mtKeima+ events were collected per condition. The raw lysosomal and cytosolic values for 20,000 events were extracted and the lysosomal/cytosolic ratio was calculated. Further details pertaining to staining and flow cytometry best practices are available in the accompanying protocol document.

\section{Western blot analysis}

Total cell lysates were prepared and run on SDS-PAGE using standard techniques. Gels were transferred onto polyvinylidene fluoride membrane using a semi-dry transfer apparatus and blocked in $5 \%$ skim milk containing TBS/T (0.1\% Tween-20). Primary and secondary antibodies were diluted in 5\% skim milk containing TBS/T and incubated at $4^{\circ} \mathrm{C}$ overnight or $1 \mathrm{~h}$ at room temperature, respectively. Primary antibodies were mouse anti-tubulin (Sigma, T9026) and rabbit anti-LC3B (Cell Signaling Technology, 3868). Secondary antibodies used for immunoblots included peroxidase-conjugated anti-rabbit and anti-mouse secondaries (Jackson ImmunoResearch Laboratories Inc., 111-035-003).

\section{Data analysis}

All data were processed in FlowJo (version 10.6) and Microsoft Excel. Graphs were generated and statistics performed in Prism 8 through a Student's $t$-test or one-way analysis of variance with Tukey multiple comparisons testing. Significance was denoted as * $(p \leq 0.05)$, ** $(p \leq 0.01){ }^{* \star *}(p \leq 0.001), * \star \star *(p \leq 0.0001)$.

\section{Results \& discussion}

In order to demonstrate the shortcomings of the current binary technique, we transiently transfected cells with mtKeima, which were then treated with 7.5 or $30 \mu \mathrm{M}$ of CCCP for $20 \mathrm{~h}$. CCCP is a common inducer of mitophagy that depolarizes mitochondria through the removal of the proton gradient [31]. We processed cells as described and performed binary gating that encompassed either 95 or $99 \%$ of the control (nonmitophagic) population, then compared our results with the widely used LC3II immunoblot assay, which measures the level of autophagic activity $[5,11]$. We determined that binary gating is capable of measuring an increase in mitophagic cells when gated to include both 95 and $99 \%$ of control events (Figure 1B). However, when employing $99 \%$ control gating, we were unable to determine a statistically different response between $7.5 \mu \mathrm{M}$ and $30 \mu \mathrm{M}$ of CCCP, even though this difference was apparent at $95 \%$ control gating and corresponded to a dose-dependent increase in LC3 lipidation observed at the same timepoint (Figure 1B \& C). Moreover, while 95\% control gating could distinguish between increasing doses of CCCP, it precluded analysis of medium and highly responsive mitophagic cells (Figure 1A, inset). The only way to include these cells in analysis with binary gating was to perform $99 \%$ control gating, which led us to conclude that this medium-mitophagic population is 'nonresponsive', even though it was obviously different than control populations (Figure 1A). Clearly, current binary gating is unable to adequately capture the variability present in mtKeima flow data.

With this in mind, we propose a consistent pipeline of cell preparation and analysis for flow cytometry using the mtKeima system. This method, termed 'ratio gating', raises the sensitivity of the assay while maximizing the reproducibility of experiments. Rather than gating on a control or mitophagic population in order to determine the mean frequency of mitophagic cells, as is the case in binary gating, we plot the lysosomal/cytosolic ratio for every cell, which corresponds to their coordinates on an mtKeima flow plot (Figures 1 $\& 2$ ). We then compare the spread of these ratios between conditions to make conclusions about the data (Figure 3B \& C). As a cell becomes more mitophagic, there will be increased fluorescence in the lysosomal channel, resulting in a higher position on the flow plot and a higher lysosomal/cytosolic ratio (Figure $3 \mathrm{C}$ ). The frequency of mitophagic cells can then be assessed by analyzing either raw or mean coordinate values for a given condition (Figure $2 A \& B$ ).

In order to demonstrate the reproducibility of ratio gating, we performed replicates of the CCCP dose curve experiment at 1-week intervals, in order to encompass both biological variability (in response to treatment and degree of mtKeima transfection) and the technical variability of the flow cytometer. Cell viability (Figure 3D) and transfection efficiency of mtKeima (Figure 3E) were similar across replicates. We found that the method is reproducible and that both the mean ratio (Figure $2 \mathrm{~A}$ ) and raw values (Figure 2B) consistently demonstrate a proportional increase in mitophagy that mirrors what is seen by LC3 immunoblotting.

Next we assessed whether ratio gating could detect changes in depolarization-induced mitophagy through the action of pharmacological inhibition. Bafilomycin-A1 (Baf) is an inhibitor of vacuolar-type H+-ATPases [32]. Treatment with Baf causes accumulation of autophagosomes as a result of impaired lysosome/autophagosome fusion and lysosome acidification. As with other cargo, recruitment and clearance of mitophagosomes within lysosomes is blunted. Notably, Baf also reduces the levels of lysosomal mtKeima when coadministered with CCCP [23]. Thus we treated cells for $20 \mathrm{~h}$ with $30 \mu \mathrm{M} \mathrm{CCCP}$ and $10 \mathrm{nM}$ Baf and analyzed the resulting flow data by both binary and ratio gating. As above, binary gating was capable of measuring an increase in mitophagic cells under CCCP treatment, when gated to include both 95 and $99 \%$ of control events (Figure 2C \& D). However, only $95 \%$ control gating was able to determine a significant reduction in mitophagy as a result of Baf. Even so, the $95 \%$ binary gate was not representative of the shifts in mitophagic populations, because the majority of measured events captured by the gate under treatment conditions were cells with low cytosolic and lysosomal fluorescence (Figure 2C). As with previous experiments, the $99 \%$ gate captured more of the middle and high mitophagic populations 
but misattributed them to the 'nonresponsive' group (Figure 2C). Ratio gating (Figure 2D) was capable of measuring the effect of Baf without the need for additional, inaccurate gating.

As ratio gating relies on extracting the coordinate information of each point, it requires specific treatment of the raw data. A detailed protocol is available.

Mitophagy is a key cellular process with wide-reaching physiological roles. While current assays are sufficient to analyze mitophagy in a cell population, single cell mitophagy assays are still in development. Recently, the advent of mtKeima flow cytometry has facilitated the precise quantification of mitophagy at a cellular level. However, as demonstrated, current binary gating techniques are lacking insofar as they require assigning biologically complex data sets into one of two categories. In comparison, our method of ratio gating facilitates reproducible analysis of single-cell mitophagy while preserving the variability present in a mitophagic population. By including a viability stain, relevant controls and best practices borrowed from multiparameter flow cytometry, we present a standardized pipeline for the analysis of mitophagy in cultured cells that can be tailored to a variety of different time points, mitophagic stimuli and cell lines.

\section{Future perspective}

It is now understood that baseline mitophagy occurs in vivo in multiple organs and tissues [6]. The presence of homeostatic levels of mitophagy requires the adoption of experimental designs and descriptive language that emphasize degrees of mitophagy, rather than binary states, in order to make comparisons at baseline and in tissues that have undergone a stressor such as exhaustive exercise [33].

Similarly, as new tools are developed to assess mitophagy in both in vitro and animal models, new analysis methodologies are also required. Recently, a novel fluorescent protein based mitophagy assay, termed mito-SRAl, has been described [34]. Similar to mtKeima, mito-SRAl can be used for quantifying lysosomal mitochondria and is compatible with flow cytometry. Unlike mtKeima, mito-SRAI is functional in fixed cell or tissue samples, providing greater flexibility over previous fluorescent systems. However, analysis of mito-SRAI flow cytometry is currently restricted to binary gating [34]. Therefore it is likely that the adoption of ratio gating will provide a more detailed interpretation of mito-SRAI data. Indeed, as flow cytometry assays and fluorescent reporter systems become more numerous and diverse in mitophagy research, it will be crucial to have clear, well-validated best practice guidelines surrounding data acquisition and processing.

\section{Author contributions}

N Winsor and S Killackey contributed equally and designed, executed and analyzed all experiments. D Philpott and S Girardin contributed to experimental design and data analysis and assisted in production of the manuscript with $\mathrm{N}$ Winsor and S Killackey.

\section{Acknowledgments}

We would like to thank D White of the Faculty of Medicine Flow Cytometry Service Team for their technical assistance and discussions throughout this project.

\section{Financial \& competing interests disclosure}

Work in the labs of D Philpott and S Girardin is supported by funding from the Canadian Institutes of Health Research. N Winsor and S Killackey are supported, respectively, by the Canadian Institutes of Health Research Doctoral Fellowship and Vanier Canada Graduate Scholarship programs. The authors declare no conflicts of interest. The authors have no other relevant affiliations or financial involvement with any organization or entity with a financial interest in or financial conflict with the subject matter or materials discussed in the manuscript apart from those disclosed.

No writing assistance was utilized in the production of this manuscript.

\section{Protocol}

The protocol for this article is listed at dx.doi.org/10.17504/protocols.io.bimhkc36

\section{Open access}

This work is licensed under the Attribution-NonCommercial-NoDerivatives 4.0 Unported License. To view a copy of this license, visit http: //creativecommons.org/licenses/by-nc-nd/4.0/

\section{References}

1. Friedman JR, Nunnari J. Mitochondrial form and function. Nature 505(7483), 335-343 (2014).

2. Ding WX, Yin XM. Mitophagy: mechanisms, pathophysiological roles, and analysis. Biol. Chem. 393(7), 547-564 (2012).

3. Springer MZ, Macleod KF. In Brief: mitophagy: mechanisms and role in human disease. J. Pathol. 240(3), $253-255$ (2016).

4. Villa E, Marchetti S, Ricci JE. No parkin zone: mitophagy without parkin. Trends Cell Biol. 28(11), 882-895 (2018).

5. Youle RJ, Narendra DP. Mechanisms of mitophagy. Nat. Rev. Mol. Cell. Biol. 12(1), 9-14 (2011).

6. McWilliams TG, Prescott AR, Montava-Garriga L et al. Basal mitophagy occurs independently of PINK1 in mouse tissues of high metabolic demand. Cell Metab. 27(2), 439-449.e435 (2018).

7. Lee JJ, Sanchez-Martinez A, Zarate AM et al. Basal mitophagy is widespread in Drosophila but minimally affected by loss of PINK1 or parkin. J. Cell Biol. 217(5), 1613-1622 (2018). 
8. Klionsky DJ, Abdelmohsen K, Abe A et al. Guidelines for the use and interpretation of assays for monitoring autophagy (3rd edition). Autophagy 12(1), 1-222 (2016).

9. Vives-Bauza C, Zhou C, Huang Y et al. PINK1-dependent recruitment of Parkin to mitochondria in mitophagy. Proc. Natl Acad. Sci. USA 107(1), 378-383 (2010).

10. Kim I, Lemasters JJ. Mitochondrial degradation by autophagy (mitophagy) in GFP-LC3 transgenic hepatocytes during nutrient deprivation. Am. J. Physiol. Cell Physiol. 300(2), C308-C317 (2011).

11. Mizushima N, Yoshimori T. How to interpret LC3 immunoblotting. Autophagy 3(6), 542-545 (2007).

12. Barth S, Glick D, Macleod KF. Autophagy: assays and artifacts. J. Pathol. 221(2), 117-124 (2010).

13. Misinzo G, Delputte PL, Nauwynck HJ. Inhibition of endosome-lysosome system acidification enhances porcine circovirus 2 infection of porcine epithelial cells. J. Virol. 82(3), 1128-1135 (2008).

14. Mindell JA. Lysosomal acidification mechanisms. Annu. Rev. Physiol. 74, 69-86 (2012).

15. Sun N, Yun J, Liu J et al. Measuring in vivo mitophagy. Mol. Cell. 60(4), 685-696 (2015).

16. Lazarou M, Sliter DA, Kane LA et al. The ubiquitin kinase PINK1 recruits autophagy receptors to induce mitophagy. Nature 524(7565), 309-314 (2015).

17. McWilliams TG, Prescott AR, Allen GF et al. mito-QC illuminates mitophagy and mitochondrial architecture in vivo. J. Cell Biol. 214(3), 333-345 (2016).

18. Dolman NJ, Chambers KM, Mandavilli B, Batchelor RH, Janes MS. Tools and techniques to measure mitophagy using fluorescence microscopy. Autophagy 9(11), 1653-1662 (2013).

19. Williams JA, Zhao K, Jin S, Ding WX. New methods for monitoring mitochondrial biogenesis and mitophagy in vitro and in vivo. Exp. Biol. Med. (Maywood) 242(8), 781-787 (2017).

20. Sun N, Malide D, Liu J, Rovira II, Combs CA, Finkel T. A fluorescence-based imaging method to measure in vitro and in vivo mitophagy using mt-Keima. Nat. Protoc. 12(8), 1576-1587 (2017).

21. Katayama H, Kogure T, Mizushima N, Yoshimori T, Miyawaki A. A sensitive and quantitative technique for detecting autophagic events based on lysosomal delivery. Chem. Biol. 18(8), 1042-1052 (2011).

22. Fang EF, Palikaras $\mathrm{K}$, Sun $\mathrm{N}$ et al. In vitro and in vivo detection of mitophagy in human cells, C. elegans, and mice. J. Vis. Exp. 129, e56301 (2017).

23. Um JH, Kim YY, Finkel T, Yun J. Sensitive measurement of mitophagy by flow cytometry using the pH-dependent fluorescent reporter mt-Keima. J. Vis. Exp. 138, e58099 (2018).

24. Lu X, Altshuler-Keylin S, Wang Q et al. Mitophagy controls beige adipocyte maintenance through a Parkin-dependent and UCP1-independent mechanism. Sci. Signal. 11(527), (2018).

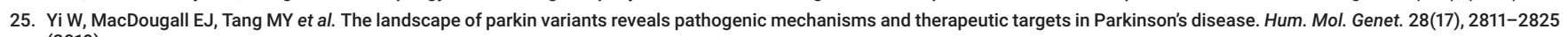
(2019).

26. Villa E, Proïcs E, Rubio-Patiño C et al. Parkin-independent mitophagy controls chemotherapeutic response in cancer cells. Cell Rep. 20(12), 2846-2859 (2017).

27. Selliah N, Eck S, Green C et al. Flow cytometry method validation protocols. Curr. Protoc. Cytom. 87(1), e53 (2019).

28. Cossarizza A, Chang HD, Radbruch A et al. Guidelines for the use of flow cytometry and cell sorting in immunological studies (second edition). Eur. J. Immunol. 49(10), 1457-1973 (2019).

29. Müller-Rischart AK, Pilsl A, Beaudette $P$ et al. The E3 ligase parkin maintains mitochondrial integrity by increasing linear ubiquitination of NEMO. Mol. Cell 49(5), 908-921 (2013).

30. Biosa A, Sanchez-Martinez A, Filograna R et al. Superoxide dismutating molecules rescue the toxic effects of PINK1 and parkin loss. Hum. Mol. Genet. 27(9), 1618-1629 (2018).

31. de Graaf AO, van den Heuvel LP, Dijkman HB et al. Bcl-2 prevents loss of mitochondria in CCCP-induced apoptosis. Exp. Cell Res. 299(2), 533-540 (2004).

32. Yoshimori T, Yamamoto A, Moriyama Y, Futai M, Tashiro Y. Bafilomycin A1, a specific inhibitor of vacuolar-type H(+)-ATPase, inhibits acidification and protein degradation in lysosomes of cultured cells. J. Biol. Chem. 266(26), 17707-17712 (1991)

33. Sliter DA, Martinez J, Hao L et al. Parkin and PINK1 mitigate STING-induced inflammation. Nature 561(7722), 258-262 (2018).

34. Katayama H, Hama H, Nagasawa K et al. Visualizing and modulating mitophagy for therapeutic studies of neurodegeneration. Cell 181(5), 1176-1187.e1116 (2020). 